\title{
Theory for Morphology Engineering of Solid Compounds (ATMESC): Facts, Predictions and Experimental Evidences
}

\author{
Abdelmohsen $\mathrm{AH}^{1-4 *}$ \\ 1 Institute of Condensed Matter and Nanosciences (IMCN), Bio-and Soft Matter, Université Catholique de Louvain, Louvain la Neuve, B-1348, Belgium \\ ${ }^{2}$ University of Grenoble Alpes, Grenoble INP-PHELMA, 3 parvis Louis Ne'el, 38016 Grenoble Cedex, France \\ ${ }^{3}$ Augsburg University, Institute of Physics, Universitätsstrass 1, 86159 Augsburg, Germany \\ ${ }^{4}$ Materials Science and Nanotechnology Department, Faculty of Postgraduate Studies for Advanced Science (PSAS), Beni-Suef University, 62511 Beni-Suef, Egypt
}

A theory for morphology engineering of solid compounds (ATMESC) that may cause a breakthrough in materials science and engineering is introduced. The ability of zinc oxide $(\mathrm{ZnO})$, and expected similar behaviour of cuprous oxide $\left(\mathrm{Cu}_{2} \mathrm{O}\right)$ and other binary compounds that exhibit $\left(\mathrm{d}^{10}\right)$ orbitals to experience morphology transition when react with phosphomolybdic acid (PMA), has initiated the idea of the ability of other solid chemical compounds to acquire polar surfaces to experience morphology transition under certain conditions. Facts, predictions and some experimental evidences are discussed which support the theory.

Abdelmohsen theory for morphology transition engineering (ATMTE) has paved the way towards a general theory that may govern the morphology transition of nearly all solid compounds under specific conditions [1]. The morphology transition of zinc oxide was explained physically by attributing the inducing of polarity to the repulsion force between electron clouds of surface ions and polyoxometalates anions (POMs anions) which induces relaxation of outer surface atoms $[1,2]$. The contribution of chemical etching was considered and supported by the ability of molybdates to form intermediate compounds like Zn-molybdates (ZM) or/and $\mathrm{Zn}$ phosphomolybdate (ZMP) (pigments) when react with zinc cations. Intermediate compound mechanism (ICM) was supposed which may involve dissolution and re-crystallization of zinc oxide to facilitate solid-state fusion [1-3].

From a point of view of a chemist, the concept of materials engineering [4] was expanded to include "controlling and designing the oriented structures of materials by rescaling their dimensions [5-7] or varying their external morphologies [8-10], favorably with functionalization [11-13], decoration [14-17], doping [18-21] or mixing [22,23] with other materials to attend the synergistic effect [24-26] which enhance their properties". There are two main methods used for nanofabrication; Top-down and bottom-up approaches. The bottom-up approach is recommended than the top-down approach, because the former can produce structures with homogenous chemical composition, and better short- and long-range ordering [1]. Figure 1 shows schematically the engineering of nanomaterials by different approaches.

The morphology evolution of zinc oxide $(\mathrm{ZnO})$ from nanorods to hybrid nanoplatelets has initiated the idea of postulating a theory (ATMTE) that was expected to govern binary compounds especially those have similar physico-chemical properties to that of zinc oxide [1]. ATMTE states that "Binary compounds especially amphoteric/ diamagnetic pure and doped metal-oxides like $\left(\mathrm{ZnO}, \mathrm{Cu}_{2} \mathrm{O}\right)$ that have appropriate energy difference between their LUMO (acid site/ cation) and HOMO (base site/anion), may experience morphology transition to various dimensions $(1 \mathrm{D}, 2 \mathrm{D}$ and $3 \mathrm{D})$ when reacts with polyoxometalates under specific conditions, with a possibility to manipulate their surface catalytic properties" [1]. ATMTE has expected similar behviour for all binary compounds that acquire $\left(\mathrm{d}^{10}\right)$ electronic configuration. This expectation still need more evidences as some binary compounds that exhibit $\left(\mathrm{d}^{10}\right)$ like $\mathrm{ZnS}$, and CdS have not responses to PMA at the same condition that was applied to $\mathrm{ZnO}$ to experience morphology transition [2]. We still believe in this prediction, and suppose that specific conditions like high PMA concentrations and high temperature may induce their surface polarity. More criticism was pointed towards ATMTE and their supposed mechanism [2]; for instance ATMTE has not predicted the morphology of engineered structures, has not mentioned the ability of $\mathrm{Zn}(\mathrm{OH})_{2}$ to experience morphology transition, has not controlled thickness of nanoplatelets, and has not predicted the ability of phosphomolybdic acid to react with $\mathrm{ZnO}$ forming intermediate compounds like $\mathrm{Zn}$-molybdates ( $\mathrm{ZM})$ or/ and $\mathrm{Zn}$-phosphomolybdate (ZMP). Moreover, they have not achieved complete morphology transition of the starting materials, and they have not sinter the final product that may enhance their physicochemical properties [1]. Further progress in work lead to postulation of a series of morphology engineering rules (MERs) that manipulates the dimension and morphology of the engineered structures [2].

On the basis of all reported articles and a wide survey, we conclude that inducing surface polarity (creating dangling bonds) is the key factor for morphology transition engineering $[1,2,27,28]$. At specific conditions, this step is followed by stabilization process that can be achieved by fusion, reconstruction or adsorption of charged species [2941]. Dangling bond can be defined as "An extremely reactive chemical bond that can be created chemically, physically or mechanically on the surface layer of solid compound" [27,42-49]. Atoms with few bonding partners acquire dangling bonds pointing outward and are known as immobilized atoms.

Dangling bond can be described as an immobilized free radical, as they are very similar to free radicals, except since they are immobilized in a solid, they are somewhat less reactive than free radicals [50]. Evidences to our theory are the ability of particles exhibiting dangling bonds to fuse with each other [1], they can react with any materials they are exposed to, such as water vapor, oxygen, hydrogen, charged species...etc. [51-56], in addition, they can reconstruct and terminate the surface when two neighboring dangling bonds bind together [5763]. Moreover, atomically precise quantum states, self-directed growth of molecular nanostructures and atomic metal wires were successfully

*Corresponding author: Ahmed Hashem Abdelmohsen, Materials Science and Nanotechnology Department, 62511 Beni-Suef, East of the Nile-PSAS, Beni-Suef University, Egypt; E-mail: ahmed.abdelmohsen@student.uni-augsburg.de

Received July 09, 2017; Accepted September 18, 2017; Published September 28, 2017

Citation: Abdelmohsen AH (2017) Theory for Morphology Engineering of Solid Compounds (ATMESC): Facts, Predictions and Experimental Evidences. J Material Sci Eng 6: 377. doi: 10.4172/2169-0022.1000377

Copyright: (c 2017 Abdelmohsen $\mathrm{AH}$. This is an open-access article distributed under the terms of the Creative Commons Attribution License, which permits unrestricted use, distribution, and reproduction in any medium, provided the original author and source are credited. 
(a)

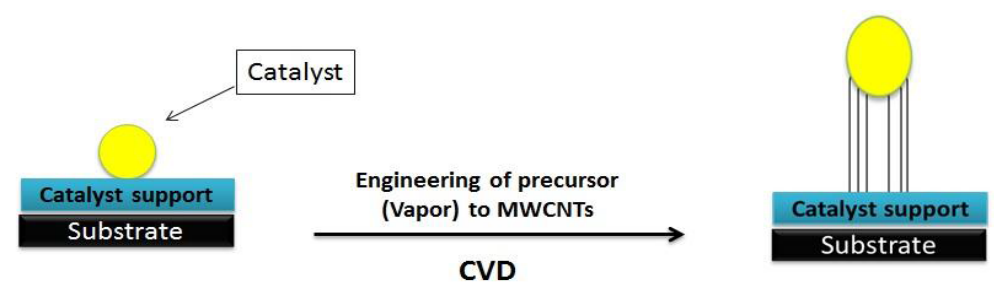

(b)

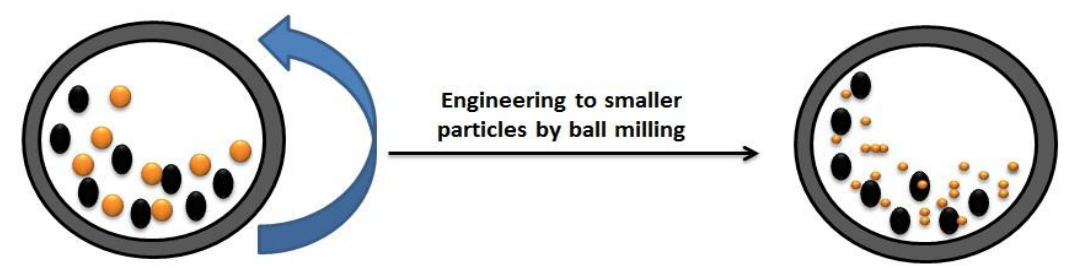

(c)



(3D)

\section{Engineering to graphene by various approaches}

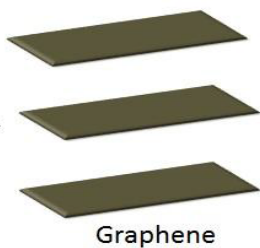

(2D)

Engineering to
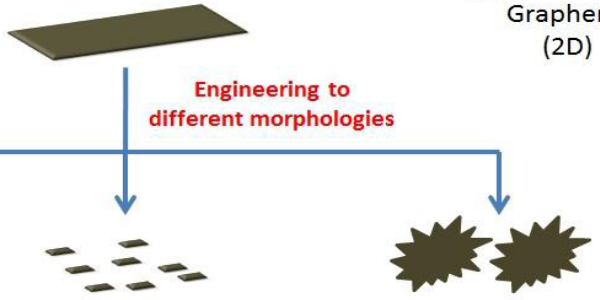

Porous Graphene

(2D)

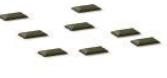

Quantum Dots

(1D)
Crumble Graphene

(3D)

Figure 1: Engineering of nanomaterials by different approaches (a) bottom-up approach, (b and c) top-down approach.

fabricated on silicon using their dangling bonds [64-66]. Whatever the way by which the dangling bonds react, they tend to minimize the surface Gibbs free energy to attain stability $[44,67,68]$.

Creating dangling bonds can take place by various ways. Firstly, we suppose chemical etching by etching-agents [69-71] like acids (e.g. phosphomolybdic acids (PMA) and reduced PMA) [1]. PMA has been used successfully to induce the polarity of $\mathrm{ZnO}$ and it is expected to induce the polarity of $\left(\mathrm{Zn}(\mathrm{OH})_{2}\right)$ which has Surface chemistry similar to that of $\mathrm{ZnO}$. POMs are expected to induce the polarity of other zinc compounds, besides other metal oxides. Chemical reagents may induce the surface polarity physically as in case of zinc oxide $(\mathrm{ZnO})$ when react with PMA [1]. This case also involves reaction of etchingagents with solid forming intermediate compounds (ZM and/or ZMP) as shown in Figure 2 [2]. Electronegativity may play role in the ability of metal oxides to acquire polar surfaces chemically [1]. Moreover, organic layers that are used for functionalization and passivation of $\mathrm{Si}$ surfaces create dangling bonds at intermediate step of reaction [72,73]. To sum up, morphology transition by chemical inducing of polarity was successfully used for engineering $1 \mathrm{D}, 2 \mathrm{D}$ and $3 \mathrm{D}$ nanostructures and ultra-thin sheets $(\sim 30 \mathrm{~nm})$ at room temperature and low concentration of PMA [1,2]. Secondly, it's a fact that; the simplest way to introduce dangling bonds physically is to create a vacancy as in case of semiconductors. For instance, pure covalent materials can acquire dangling bonds on their surfaces by removal of the central atom [27].
This leads to rupture of four covalent bonds, i.e. the creation of four dangling bonds as shown schematically in Figure 3b. It is supposed that surface bombardment by particles/ions may be used for this reason. Imaginary set-up for physical inducing of polarity is shown in Figure 3a.

Dangling bonds can be also induced by light as in case of amorphous silica [74-80]. Moreover, physical etching of other solid compounds rather than covalent compounds may create dangling bonds on their surfaces. We suppose that some charged species like $\left(\mathrm{O}^{2-}\right)$ or molecules can bind nanoparticles together which cause fusion and morphology transition as shown in Figure 3c [65]. Furthermore, ability of engineering quantum layers (atomic wires) [66] that may be followed by engineering of multi-atomic layers may support the validity of this theory (ATMESC) as shown in Figure 3c.

Thirdly, breaking large particles mechanically creates particles with polar surface. In other words, solid compounds under mechanical force experience bond cleavage which leads to smaller particles with polar surfaces [28,81-89]. Ball milling of metal oxides (e.g. $\mathrm{ZnO}$ ) is expected to induce the surface polarity as shown in Figure 4. Inert atmosphere condition is supposed in case of solid compounds that are etched physically or mechanically to avoid reconstruction [57-59] or adsorption of charged species [51,52]. This approach may be used for minimizing size before applying chemical or physical treatment to induce polarity (e.g., milling commercial $\mathrm{ZnO}$ (microscale) before 


\section{$\mathrm{Zn}_{n} \mathrm{PMO}_{\mathrm{x}} \mathrm{O}_{\mathrm{y}} \mathrm{Z}^{-} \mathrm{Zn}$-phosphomolybdate $\mathrm{Zn}_{\mathrm{n}} \mathrm{Mo}_{\mathrm{x}} \mathrm{O}_{\mathrm{y}}{ }^{\mathrm{Z}-} \mathrm{Zn}$-molybdate $\mathrm{POM}-\mathrm{POM}$ anions}

(a) Physical Explanation:
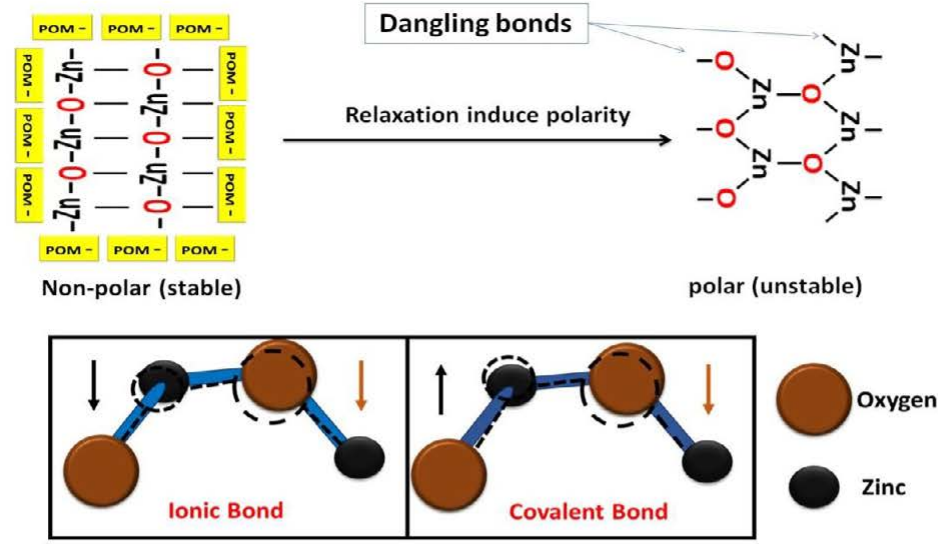

(b) Chemical Explanation:

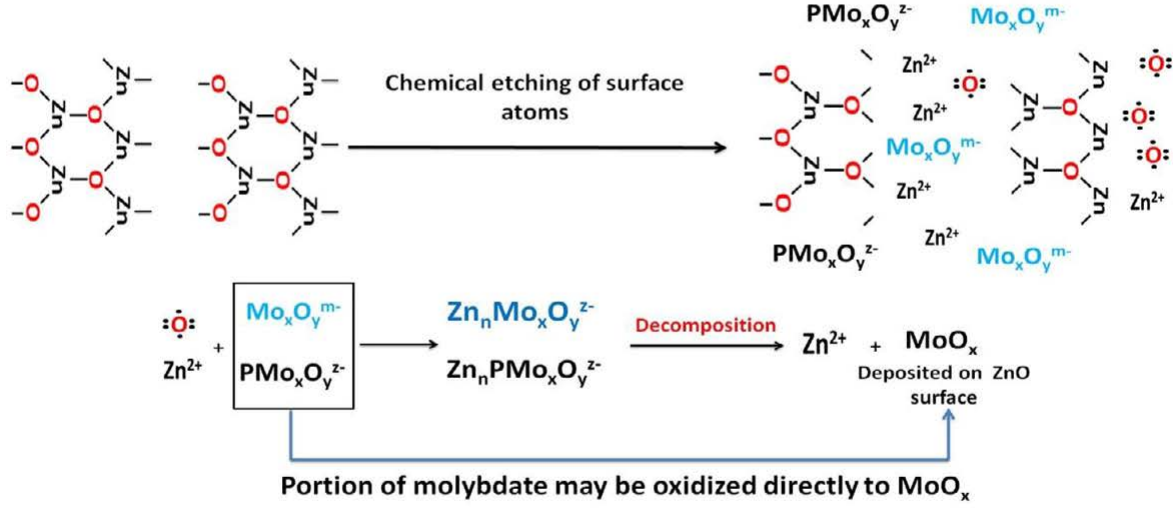

(c) Fusion Process:

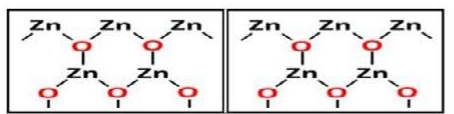

Low dimension (1D)

[Short-chain fusion]

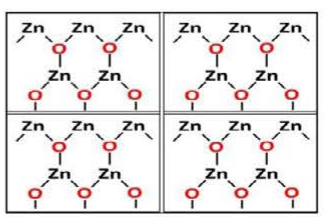

High dimensions (2D, 3D)

[Long-chain fusion]

Figure 2: Creating dangling bonds (inducing surface polarity) chemically for zinc oxide; (a) physical explanation, (b) chemical explanation, and (c) fusion process.

immersing in PMA solution to induce morphology transition) $[1,2]$. Milling can be used directly for morphology transition without further treatment, by dividing bulk particles to small structures [82,84].

After inducing the polarity of solid compounds, we suppose treating them in a certain medium for attaining stability by different ways (e.g., solution as a medium in presence of external cations, anions, chemical reagent to induce fusion process) [1,2]. Solid compounds with active sites like $\mathrm{ZnO}$ may catalyze deposition of molybdenum oxides over their surfaces, if they dispersed in POMs solution [1,2]. Other medium may be suggested that may keep dangling bonds without passivation. In-situ TEM analysis for these experiments is suggested to investigate the fusion of nanoparticles [90,91]. Properties of the dangling bonds may govern the feasibility of morphology transition process [92-94]. Under specific conditions, physical and mechanical treatment is enough for inducing morphology transition, i.e., we do not need further treatment as mentioned above. For instance, physical treatment of solid compounds by bombardment with highly energetic particles/rays is expected to cause ions redistribution which may cause morphology transition. In case of mechanical treatment, highenergy ball milling cause also cations redistribution as reported here [95]. In addition, $\mathrm{ZnO}$ nanowires were engineered by ball milling and annealing methods [96].

Herein, on the basis of a theory for morphology transition engineering Theory (ATMTE) [1], suggestions, experimental studies and scientific facts; we introduce a new theory that may be applied to most of solid compounds; Abdelmohsen Theory for Morphology 
(a)

(b)
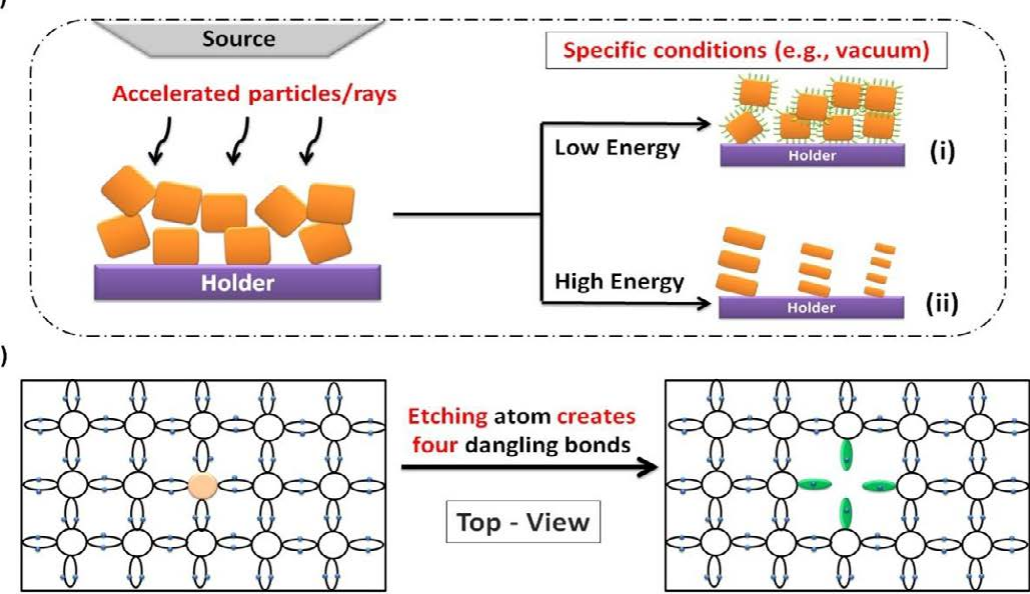

(c)

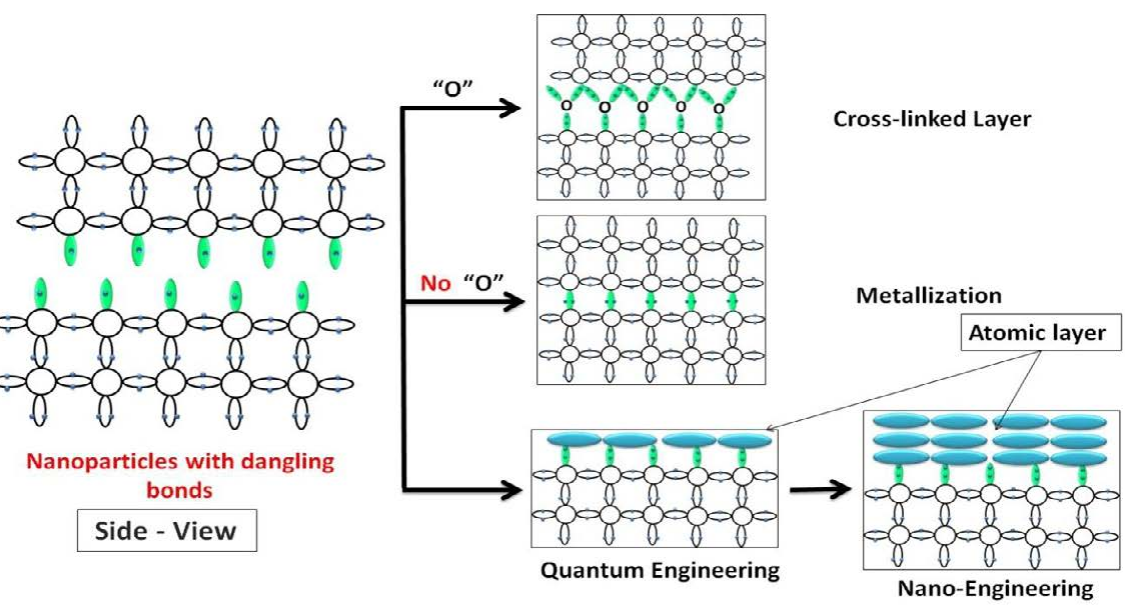

Figure 3: Shows (a) experimental set-up for physical creation of dangling bonds, (b) top-view for etching surface atom of semiconductor (e.g. Silicon) to create four dangling bonds, and (c) supposed engineering processes for semiconductor nanoparticles by fabricating atomic wires on surface.

Engineering of Solid Compounds (ATMESC): which states that "Most solid compounds may experience morphology transition by inducing their surface polarity (creating dangling bonds); chemically, physically or mechanically. For instance, chemically we can induce polarity of amphoteric compounds which include diamagnetic cations $\left(d^{10}\right)$ within their structure (e.g., doped/pure $\mathrm{ZnO}$ or $\mathrm{Cu}_{2} \mathrm{O}$ and their hydroxides, compounds and composites) with a possibility to manipulate the engineered morphologies, and surface catalytic activity depending on the morphology engineering rules (MERs)".

In order to simplify the idea, Figure 5 a illustrates schematically the growth mechanism of nanoparticles by bottom-up approach $[97,98]$; starting by mixing the reactants under specific conditions, passing by nucleation of seeds to the formation of the active unstable state (AUS) (polar state) in which the particles still keep growth by binding their dangling bonds to other species. Particles may reconstruct to terminate growth in preferred orientation according to specific conditions and rules $[1,99]$. The final solid product will have terminated surfaces (no dangling bonds). The idea is to allow these solid compounds to acquire the surface properties of active unstable state (AUS). By this way, they will have the ability to fuse to each other, reconstruct to preferred morphologies, or adsorb charged species as shown in Figure 5b. Immersing polar particles in ionic solution e.g., $\mathrm{ZnO}$ polar particles in $\mathrm{ZnSO}_{4}$ or $\mathrm{ZnCl}_{2}$ solution may have influence on the morphology of engineered structures as external cations will contribute to morphology engineering.

It is supposed that, there will be selection criteria for the materials to acquire dangling bonds by each suggested methods, such as size, and chemical composition. Hybrid and pure nanostructures (1D, $2 \mathrm{D}$, and $3 \mathrm{D}$ ) of $\mathrm{ZnO}$ that were engineered by chemical method is expected to have potential use in catalysis, energy, biomedical and other applications; especially few and one-atom thick layer structures (Zn-oxidene) $[1,2,100]$. Moreover, many papers have been reported about morphology evolution and surface decoration and their effect on the physio-chemical properties of materials [101,102]. In addition, carbonaceous materials like graphene and carbon nanotubes may follow this theory (ATMESC) [82,103-105]. Progress in scientific research will facilitate the way to introduce more evidences for this theory. This theory is expected to be criticized, or corrected like most theories that have been suggested before [106,107].

\section{Conclusions}

The possibility for inducing surface polarity of solid compounds by various ways (chemically, physically, and mechanically) was introduced. 
(a)

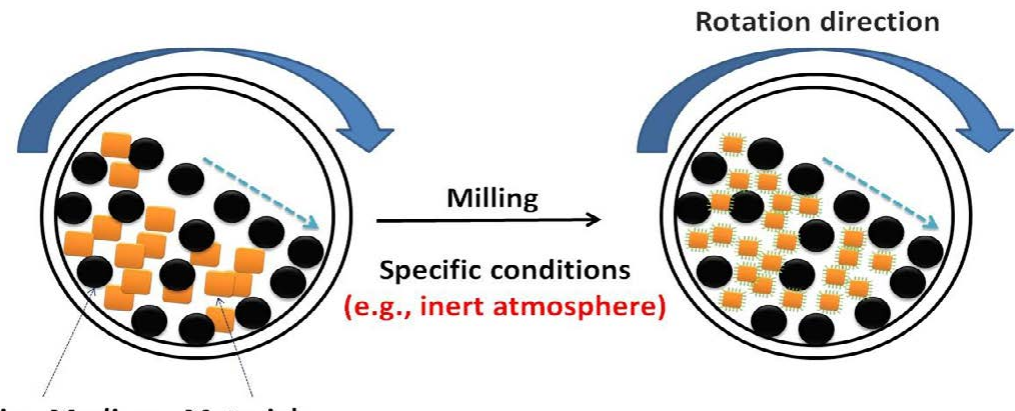

Grinding Medium Materials

(b)

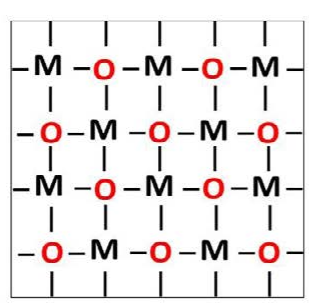

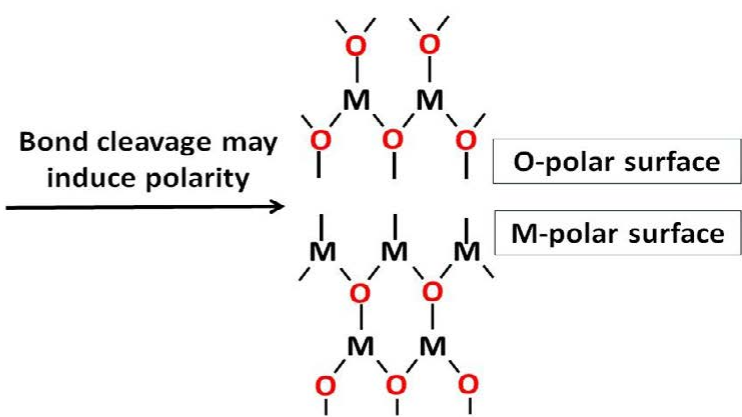

Figure 4: Shows (a) mechanical creation of dangling bonds (inducing polarity) by ball milling in inert atmosphere, and (b) bond cleavage of metal oxides particle (e.g. $\mathrm{ZnO})$.

(a)

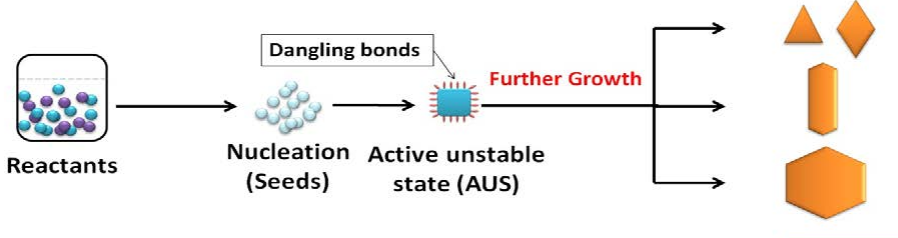

(b)

$\stackrel{\text { (i) Fusion }}{\longrightarrow}$

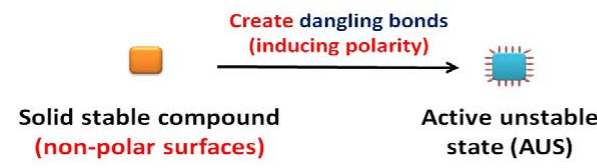

(ii) Reconstruction to

preferred morphologies

(non-polar surfaces)

state (AUS)

(iii) Surface reconstruction

Atomic layer

(iv) Nano/quantum engineering

Figure 5: Shows (a) supposed growth mechanism of various nanostructures (metal oxides) in solution, and (b) inducing the polarity of solid stable compound

This suggestion supports the theory (ATMESC) which paves the way to manipulate pure and doped $(1 \mathrm{D}, 2 \mathrm{D}$, and $3 \mathrm{D})$ nanostructures that have potential applications in nearly all fields of science. This theory is expected to cause breakthrough in materials science and engineering if more experimental evidences are introduced. In addition, it will be used for explaining the morphology evolution phenomenons for nearly all solid compounds.

\section{Acknowledgment}

Prof. MM Khalil (PhD-UCLA) deserves acknowledgement as he has named the author as "a theoretically talented student". Thanks to Ass. Prof. Ali Q Selim for informing me about his novel work on morphology evolution by surface bombardment. Thanks to Erasmus Mundus Organization for Funding Mr. Ahmed Abdelmohsen to study MSc. (FAME program-2015/2017) at Augsburg UNI, Grenoble INP-PHELMA and Université Catholique de Louvain. Thanks to the Egyptian team at Beni-Suef University \& National Research Centre. Thanks to the European team at Grenoble PHELMA-INP and Université Catholique de Louvain. 


\section{References}

1. Abdelmohsen AH, El Rouby WM, Ismail N, Farghali AA (2017) Morphology Transition Engineering of $\mathrm{ZnO}$ Nanorods to Nanoplatelets Grafted Mo8O23$\mathrm{MoO} 2$ by Polyoxometalates: Mechanism and Possible Applicability to other Oxides. Scientific Reports, 7.

2. Abdelmohsen A, Bouchet R, and Vlad A, (2017) Morphology Transition Engineering of 1D, 2D, and 3D Hybrid Nanostructures. 9th EMMC FAME Research Workshop, Liège University, Research gate.

3. Karekar SE, Bhanvase BA, Sonawane SH, Deosarkar MP, Pinjari DV, et al. (2015) Synthesis of zinc molybdate and zinc phosphomolybdate nanopigments by an ultrasound assisted route: advantage over conventional method. Chemical Engineering and Processing: Process Intensification 87: 51-59.

4. Callister WD, Rethwisch DG (2011) Materials science and engineering (Vol. 5). NY: John Wiley \& Sons.

5. Romo-Herrera JM, Terrones M, Terrones H, Dag S, Meunier V (2007) Covalen 2D and 3D networks from 1D nanostructures: designing new materials. Nano Letters 7: $570-576$

6. Joshi RK, Schneider JJ (2012) Assembly of one dimensional inorganic nanostructures into functional $2 \mathrm{D}$ and $3 \mathrm{D}$ architectures. Synthesis, arrangement and functionality. Chemical Society Reviews 41: 5285-5312.

7. Huang X, Li J, Zhang Y, Mascarenhas A (2003) From 1D chain to 3D network: tuning hybrid II-VI nanostructures and their optical properties. Journal of the American Chemical Society 125: 7049-7055

8. Yerra S, Supriya S, Das SK (2013) Reversible morphological transition between nano-rods to micro-flowers through micro-hexagonal crystals in a sonochemical synthesis based on a polyoxovanadate compound. Inorganic Chemistry Communications 35: 54-57.

9. Xiong S, Yuan C, Zhang X, Xi B, Qian Y (2009) Controllable synthesis of mesoporous $\mathrm{Co} 3 \mathrm{O} 4$ nanostructures with tunable morphology for application in supercapacitors. Chemistry-A European Journal 15: 5320-5326.

10. Khomutov GB, Kislov VV, Gainutdinov RV, Gubin SP, Obydenov AY et al. (2003) The design, fabrication and characterization of controlledmorphology nanomaterials and functional planar molecular nanocluster-based nanostructures. Surface Science 532: 287-293.

11. Reddy KR, Sin BC, Ryu KS, Kim JC, Chung H, et al. (2009) Conducting polymer functionalized multi-walled carbon nanotubes with noble metal nanoparticles: synthesis, morphological characteristics and electrical properties. Synthetic Metals 159: $595-603$

12. Huh S, Wiench JW, Yoo JC, Pruski M, Lin VSY (2003) Organic functionalization and morphology control of mesoporous silicas via a co-condensation synthesis method. Chemistry of Materials 15: 4247-4256.

13. Liu X, Zhang J, Wang L, Yang T, Guo X, et al. (2011) 3D hierarchically porous $\mathrm{ZnO}$ structures and their functionalization by Au nanoparticles for gas sensors. Journal of Materials Chemistry 21: 349-356.

14. Wang L, Kang Y, Wang Y, Zhu B, Zhang S, et al. (2012) CuO nanoparticle decorated $\mathrm{ZnO}$ nanorod sensor for low-temperature $\mathrm{H} 2 \mathrm{~S}$ detection. Materials Science and Engineering: C 32: 2079-2085.

15. Rashid TR, Phan DT, Chung GS (2013) A flexible hydrogen sensor based on $\mathrm{Pd}$ nanoparticles decorated $\mathrm{ZnO}$ nanorods grown on polyimide tape. Sensors and Actuators B: Chemical 185: 777-784.

16. Rakshit T, Mondal SP, Manna I, Ray SK (2012) CdS-decorated ZnO nanorod heterostructures for improved hybrid photovoltaic devices. ACS Applied Materials \& Interfaces 4: 6085-6095.

17. Hu T, Chen L, Deng Z, Chen Y (2015) Amphiphilic fullerenes modified 1D ZnO arrayed nanorods-2D graphene hybrids as cathode buffer layers for inverted polymer solar cells. Journal of Materials Chemistry A 3: 10890-10899.

18. Gong $\mathrm{H}$, Hu JQ, Wang JH, Ong CH, Zhu FR (2006) Nano-crystalline Cu-doped $\mathrm{ZnO}$ thin film gas sensor for CO. Sensors and Actuators B: Chemical 115: 247-251.

19. Ronning C, Gao PX, Ding Y, Wang ZL, Schwen D (2004) Manganese-doped ZnO nanobelts for spintronics. Applied Physics Letters 84: 783-785.

20. Rani S, Roy SC, Bhatnagar MC (2007) Effect of Fe doping on the gas sensing properties of nano-crystalline SnO 2 thin films. Sensors and Actuators B: Chemical 122: 204-210.
21. Wei D, Liu Y, Wang Y, Zhang H, Huang L, et al. (2009) Synthesis of N-doped graphene by chemical vapor deposition and its electrical properties. Nano Letters 9: 1752-1758.

22. Jongsomjit B, Kittiruangrayub S, Praserthdam P (2007) Study of cobalt dispersion onto the mixed nano-SiO2-ZrO2 supports and its application as a catalytic phase. Materials Chemistry and Physics 105: 14-19.

23. Valand J, Parekh H, Friedrich HB (2013) Mixed Cu-Ni-Co nano-metal oxides: a new class of catalysts for styrene oxidation. Catalysis Communications 40 : 149-153.

24. Xu J, Wang K, Zu SZ, Han BH, Wei Z (2010) Hierarchical nanocomposites of polyaniline nanowire arrays on graphene oxide sheets with synergistic effect for energy storage. ACS Nano 4: 5019-5026.

25. Sarkar D, Xie X, Kang J, Zhang H, Liu W, et al. (2015) Functionalization of transition metal dichalcogenides with metallic nanoparticles: implications for doping and gas-sensing. Nano Letters 15: 2852-2862.

26. Lan Q, Yang F, Zhang S, Liu S, Xu J, et al. (2007) Synergistic effect of silica nanoparticle and cetyltrimethyl ammonium bromide on the stabilization of $\mathrm{O} / \mathrm{W}$ emulsions. Colloids and Surfaces A: Physicochemical and Engineering Aspects 302: 126-135

27. Lannoo M (1990) The role of dangling bonds in the properties of surfaces and interfaces of semiconductors. Revue de Physique Appliquée 25: 887-894.

28. Kung HH (1989) Transition metal oxides: surface chemistry and catalysis (Vol. 45). Elsevier.

29. Wander A, Schedin F, Steadman P, Norris A, McGrath R, et al. (2001) Stability of polar oxide surfaces. Physical Review Letters 86: 3811.

30. Wander A, Harrison NM (2001) The stability of polar oxide surfaces: The interaction of $\mathrm{H} 2 \mathrm{O}$ with $\mathrm{ZnO}(0001)$ and $\mathrm{ZnO}(0001)$. The Journal of Chemical Physics 115: 2312-2316

31. Chang SC, Mark $P$ (1974) The crystallography of the principal non-polar (1120) and (1010) surfaces of zinc oxide. Surface Science 45: 721-727.

32. Dulub O, Diebold U, Kresse G (2003) Novel stabilization mechanism on pola surfaces: ZnO (0001)-Zn. Physical Review Letters 90: 016102.

33. Duke CB, Lubinsky AR, Lee BW, Mark P (1976) Atomic geometry of cleavage surfaces of tetrahedrally coordinated compound semiconductors. Journal of Vacuum Science and Technology 13: 761-768.

34. Kresse G, Dulub O, Diebold U (2003) Competing stabilization mechanism for the polar ZnO (0001)-Zn surface. Physical Review B 68: 245409.

35. Goniakowski J, Noguera C (2002) Microscopic mechanisms of stabilization of polar oxide surfaces: Transition metals on the MgO (111) surface. Physical Review B 66: 085417.

36. Hesper R, Tjeng LH, Heeres A, Sawatzky GA (2000) Photoemission evidence of electronic stabilization of polar surfaces in K 3 C 60 . Physical Review B 62 : 16046.

37. Staemmler V, Fink K, Meyer B, Marx D, Kunat M, et al. (2003) Stabilization of polar $\mathrm{ZnO}$ surfaces: Validating microscopic models by using $\mathrm{CO}$ as a probe molecule. Physical Review Letters 90: 106102.

38. Pandey KC (1982) Reconstruction of semiconductor surfaces: Buckling, ionicity, and $\pi$-bonded chains. Physical Review Letters 49: 223.

39. Noguera C (2000) Polar oxide surfaces. Journal of Physics: Condensed Matter 12: R367.

40. Wang ZL, Kong XY, Ding Y, Gao P, Hughes WL, et al. (2004) Semiconducting and piezoelectric oxide nanostructures induced by polar surfaces. Advanced Functional Materials 14: 943-956.

41. Lauritsen JV, Porsgaard S, Rasmussen MK, Jensen MC, Bechstein R, et al (2011) Stabilization principles for polar surfaces of ZnO. ACS Nano 5: $5987-$ 5994.

42. Mariano AN, Hanneman RE (1963) Crystallographic polarity of ZnO crystals. Journal of Applied Physics 34: 384-388.

43. Hadis M, Ümit Ö (2009) Zinc Oxide: Fundamentals, Materials and Device Technology.

44. Tang C, Spencer MJ, Barnard AS (2014) Activity of ZnO polar surfaces: an 
insight from surface energies. Physical Chemistry Chemical Physics 16: 22139-22144.

45. Heiland G, Kunstmann P (1969) Polar surfaces of zinc oxide crystals. Surface Science 13: 72-84.

46. Hüpkes J, Owen JI, Pust SE, Bunte E (2012) Chemical Etching of Zinc Oxide for Thin-Film Silicon Solar Cells. ChemPhysChem 13: 66-73.

47. Gatos HC (1961) Dangling bonds in III-V compounds. Journal of Applied Physics 32: 1232-1234.s

48. Yates Jr JT (1991) Surface chemistry of silicon-the behaviour of dangling bonds. Journal of Physics: Condensed Matter 3(S): S143.

49. Pitters JL, Piva PG, Tong X, Wolkow RA (2003) Reversible passivation of silicon dangling bonds with the stable radical TEMPO. Nano Letters 3: 14311435.

50. Wang D, Buriak JM (2006) Trapping silicon surface-based radicals. Langmuir 22: 6214-6221.

51. Cartier E, Stathis JH, Buchanan DA (1993) Passivation and depassivation of silicon dangling bonds at the $\mathrm{Si} / \mathrm{SiO} 2$ interface by atomic hydrogen. Applied Physics Letters 63: 1510-1512.

52. Weber JR, Janotti A, Rinke P, Van de Walle CG (2007) Dangling-bond defects and hydrogen passivation in germanium. Applied Physics Letters 91: 142101.

53. Johnson NM, Biegelsen DK, Moyer MD (1982) Deuterium passivation of grainboundary dangling bonds in silicon thin films. Applied Physics Letters 40: 882-884.

54. Stesmans A, Afanas' ev VV (2003) Si dangling-bond-type defects at the interface of (100) Si with ultrathin HfO 2. Applied Physics Letters 82: 40744076 .

55. Afanas' ev VV, Fedorenko YG, Stesmans A (2005) Interface traps and dangling-bond defects in (100) Ge/Hf O 2. Applied Physics Letters 87: 032107.

56. Koskinen P, Malola S, Häkkinen H (2008) Self-passivating edge reconstructions of graphene. Physical Review Letters 101: 115502.

57. Starke U, Schardt J, Bernhardt J, Franke M, Reuter K, et al. (1998) Nove reconstruction mechanism for dangling-bond minimization: Combined method surface structure determination of $\mathrm{SiC}(111)-(3 \times 3)$. Physical Review Letters 80: 758.

58. Ajayan PM, Ravikumar V, Charlier JC (1998) Surface reconstructions and dimensional changes in single-walled carbon nanotubes. Physical Review Letters 81: 1437

59. Binnig G, Rohrer H, Gerber C, Weibel E (1983) $7 \times 7$ reconstruction on Si (111) resolved in real space. Physical Review Letters 50: 120.

60. Kern G, Hafner J, Kresse G (1996) Atomic and electronic structure of diamond (111) surfaces I. Reconstruction and hydrogen-induced de-reconstruction of the one dangling-bond surface. Surface science 366: 445-463.

61. Harrison WA (1976) Surface reconstruction on semiconductors. Surface Science 55: 1-19.

62. Kaxiras E (1990) Effect of surface reconstruction on stability and reactivity of Si clusters. Physical Review Letters 64: 551.

63. Chadi DJ (1987) Atomic structure of GaAs (100)- $(2 \times 1)$ and $(2 \times 4)$ reconstructed surfaces. Journal of Vacuum Science \& Technology A: Vacuum, Surfaces, and Films 5: 834-837.

64. Schofield SR, Studer P, Hirjibehedin CF, Curson NJ, Aeppli G, et al. (2013) Quantum engineering at the silicon surface using dangling bonds. Nature communications 4: 1649 .

65. Lopinski GP, Wayner DDM, Wolkow RA (2000) Self-directed growth of molecular nanostructures on silicon. Nature 406: 48.

66. Hashizume T, Heike S, Lutwyche MI, Watanabe S, Nakajima K, et al. (1996) Interaction of $\mathrm{Ga}$ adsorbates with dangling bonds on the hydrogen terminated $\mathrm{Si}(100)$ surface. Japanese journal of applied physics 35(8B): L1085

67. Santos E, Schmickler W (2004) Changes in the surface energy during the reconstruction of $\mathrm{Au}(100)$ and $\mathrm{Au}$ (111) electrodes. Chemical physics letters 400: 26-29.

68. Jia M, Lai Y, Tian Z, Liu Y (2008) Calculation of the surface free energy of fcc copper nanoparticles. Modelling and Simulation in Materials Science and Engineering 17: 015006.
69. Beverskog B, Puigdomenech I (1997) Revised pourbaix diagrams for zinc at 25-300 C. Corrosion Science 39: 107-114.

70. Mariano AN, Hanneman RE (1963) Crystallographic polarity of ZnO crystals. Journal of Applied Physics 34: 384-388.

71. Price PB, Walker RM (1962) Chemical Etching of Charged-Particle Tracks in Solids. Journal of Applied Physics 33: 3407-3412.

72. Hartig P, Dittrich T, Rappich J (2002) Surface dipole formation and non-radiative recombination at $\mathrm{p}-\mathrm{Si}(111)$ surfaces during electrochemical deposition of organic layers. Journal of Electroanalytical Chemistry 524: 120-126.

73. Rappich J, Hartig P, Nickel NH, Sieber I, Schulze S, et al. (2005) Stable electrochemically passivated $\mathrm{Si}$ surfaces by ultra-thin benzene-type layers. Microelectronic Engineering 80: 62-65.

74. Morigaki K (1988) Microscopic mechanism for the photo-creation of dangling bonds in a-Si: H. Japanese journal of applied physics 27(2R): 163.

75. Warren WL, Robertson J, Kanicki J (1993) Si and N dangling bond creation in silicon nitride thin films. Applied Physics Letters 63: 2685-2687.

76. Hirabayashi I, Morigaki K, Nitta S (1980) New evidence for defect creation by high optical excitation in glow discharge amorphous silicon. Japanese Journal of Applied Physics 19: 357

77. Dersch H, Stuke J, Beichler J (1981) Light-induced dangling bonds in hydrogenated amorphous silicon. Applied Physics Letters 38: 456-458.

78. Ishikawa K, Okigawa M, Ishikawa Y, Samukawa S, Yamasaki S (2005) In vacuo measurements of dangling bonds created during Ar-diluted fluorocarbon plasma etching of silicon dioxide films. Applied Physics Letters 86: 264104.

79. Warren WL, Seager CH, Robertson J, Kanicki J, Poindexter EH (1996) Creation and properties of nitrogen dangling bond defects in silicon nitride thin films. Journal of The Electrochemical Society 143: 3685-3691.

80. Morigaki K, Hikita H, Yamaguchi M, Fujita $Y$ (1998) The structure of dangling bonds having hydrogen at a nearby site in a-Si: $\mathrm{H}$. Journal of non-crystalline solids 227: 338-342.

81. Ong TS, Yang $H(2000)$ Effect of atmosphere on the mechanical milling of natural graphite. Carbon 38: 2077-2085.

82. León V, Quintana M, Herrero MA, Fierro JL, de la Hoz A, et al. (2011) Fewlayer graphenes from ball-milling of graphite with melamine. Chemical Communications 47: 10936-10938.

83. Takacs $L$ (2002) Self-sustaining reactions induced by ball milling. Progress in Materials Science 47: 355-414.

84. Pierard N, Fonseca A, Konya Z, Willems I, Van Tendeloo G, et al. (2001) Production of short carbon nanotubes with open tips by ball milling. Chemical Physics Letters 335: 1-8.

85. Basset D, Matteazzi P, Miani F (1993) Designing a high energy ball-mill for synthesis of nanophase materials in large quantities. Materials Science and Engineering: A 168: 149-152.

86. Wu JM (2001) Nano-sized amorphous alumina particles obtained by ball milling $\mathrm{ZnO}$ and Al powder mixture. Materials Letters 48: 324-330.

87. Giri PK, Bhattacharyya S, Singh DK, Kesavamoorthy R, Panigrahi BK, et al. (2007) Correlation between microstructure and optical properties of $\mathrm{ZnO}$ nanoparticles synthesized by ball milling. Journal of Applied Physics 102 : 093515

88. Bid S, Pradhan SK (2003) Preparation of zinc ferrite by high-energy ball-milling and microstructure characterization by Rietveld's analysis. Materials Chemistry and Physics 82: 27-37

89. Hindmarch AT, Parkes DE, Rushforth AW (2012) Fabrication of metallic magnetic nanostructures by argon ion milling using a reversed-polarity planar magnetron ion source. Vacuum 86: 1600-1604.

90. Anton R, Schneidereit I (1998) In situ TEM investigations of dendritic growth of Au particles on HOPG. Physical Review B 58: 13874

91. Ritchie C, Cooper GJ, Song YF, Streb C, Yin H, et al. (2009) Spontaneous assembly and real-time growth of micrometre-scale tubular structures from polyoxometalate-based inorganic solids. Nature Chemistry 1: 47-52.

92. Akiyama T, Nakamura K, Ito T (2006) Structural stability and electronic 
Citation: Abdelmohsen AH (2017) Theory for Morphology Engineering of Solid Compounds (ATMESC): Facts, Predictions and Experimental Evidences. J Material Sci Eng 6: 377. doi: 10.4172/2169-0022.1000377

Page 8 of 8

structures of InP nanowires: Role of surface dangling bonds on nanowire facets. Physical Review B 73: 235308

93. Biswas R, Wang CZ, Chan CT, Ho KM, Soukoulis CM (1989) Electronic structure of dangling and floating bonds in amorphous silicon. Physical Review Letters 63: 1491

94. Street RA (1982) Trapping parameters of dangling bonds in hydrogenated amorphous silicon. Applied Physics Letters 41: 1060-1062.

95. Fatemi DJ, Harris VG, Browning VM, Kirkland JP (1998) Processing and cation redistribution of $M n Z n$ ferrites via high-energy ball milling. Journal of Applied Physics 83: 6867-6869.

96. Glushenkov AM, Zhang HZ, Zou J, Lu GQ, Chen Y (2007) Efficient production of $\mathrm{ZnO}$ nanowires by a ball milling and annealing method. Nanotechnology 18 : 175604

97. Kuriakose S, Satpati B, Mohapatra S (2014) Enhanced photocatalytic activity of Co doped $\mathrm{ZnO}$ nanodisks and nanorods prepared by a facile wet chemical method. Physical Chemistry Chemical Physics 16: 12741-12749.

98. Wang ZL (2004) Zinc oxide nanostructures: growth, properties and applications. Journal of Physics: Condensed Matter 16: R829.

99. Chang S, and Mark P, (1974) The crystallography of the principal non-polar (1120) and (1010) surfaces of zinc oxide Surf Sci 45: 72.

100. Abdelmohsen A, Vlad A (2017) A Facile Synthesis of Exfoliated Graphite like
Hybrid Zinc Oxide at Room Temperature: First Step towards Metal-oxidene. Ph.D. Students' Day, UCLouvain (IMCN) - Research Gate.

101. Hoseinzadeh S, Ghasemiasl R, Bahari A, Ramezani AH (2017) n-type WO semiconductor as a cathode electrochromic material for ECD devices. Journal of Materials Science: Materials in Electronics 1-7.

102. Hoseinzadeh S, GhasemiasI R, Bahari A, Ramezani AH (2017) The injection of $\mathrm{Ag}$ nanoparticles on surface of WO3 thin film: enhanced electrochromic coloration efficiency and switching response. Journal of Materials Science: Materials in Electronics 28: 14855-14863.

103. Joselevich E (2004) Electronic structure and chemical reactivity of carbon nanotubes: a chemist's view. ChemPhysChem 5: 619-624

104. Akhukov MA, Fasolino A, Gornostyrev YN, Katsnelson MI (2012) Dangling bonds and magnetism of grain boundaries in graphene. Physical Review $B$ 85: 115407.

105. Ajayan P, Ravikumar V, and Charlier J, (1998) Surface Reconstructions and Dimensional Changes in Single-Walled Carbon Nanotubes. Phys Rev Lett 81: 1437

106. Di Sia P, Dallacasa V (2011) Anomalous charge transport: a new "time domain" generalization of the Drude model. Plasmonics 6: 99-104.

107. Sigmund P (1996) Low-speed limit of Bohr's stopping-power formula. Physical Review A 54: 3113 